\title{
Very early Charcot spinal arthropathy associated with forward bending after spinal cord injury: a case report
}

\author{
Benjamin A. Abramoff ${ }^{1}$. Veronica L. Sudekum² - Lisa-Ann Wuermser ${ }^{3}$ - Faiz U. Ahmad ${ }^{4}$
}

Received: 29 November 2018 / Revised: 25 January 2019 / Accepted: 30 January 2019

(c) International Spinal Cord Society 2019

\begin{abstract}
Introduction Charcot spinal arthropathy (CSA) is an uncommon clinical entity following spinal cord injury (SCI). It is characterized by progressive cartilaginous and bony destruction and is felt to be due to loss of proprioceptive and nociceptive feedback from the spine. CSA is typically diagnosed many years following SCI and has the potential to lead to progressive neurologic decline if left untreated.

Case presentation We describe the case of a 49-year-old male who fell approximately thirty feet from a ladder and sustained a fracture/dislocation at T3-4 and T8-9 resulting in a T4 ASIA A SCI. He underwent T2-T12 posterior spinal stabilization and, within 1 year and 2 months of initial injury, developed an unusual back protuberance, decreased spasticity, and change in bladder function. The patient's imaging and physical exam were consistent with CSA.

Discussion This case is notable in two respects. First, this is one of the earliest cases of CSA identified in the literature. Although CSA is generally considered a late complication of SCI, CSA should be placed in the differential for all individuals with spinal cord presenting with clinical findings typical of CSA. Second, this case was associated with unsupervised attempts to improve range of motion (ROM) in a SCI patient with a fused spine. The association of unsupervised stretching and CSA has not been previously described.
\end{abstract}

\section{Introduction}

Although neuropathic joints typically affect peripheral joints, reported cases involving the spine date back to at least 1884 [1]. When the condition occurs in the spine, it is known by several names including "neuropathic spinal arthropathy," "spinal neural arthropathy," "spinal neuropathic joints," "Charcot spine," and "Charcot spinal

$\triangle$ Benjamin A. Abramoff

Benjamin.Abramoff@uphs.upenn.edu

1 Perelman School of Medicine at the University of Pennsylvania, 1800 Lombard St. First Floor, Philadelphia, PA 19146, USA

2 Emory Physical Medicine and Rehabilitation, 1441 Clifton Road, Atlanta, GA 30322, USA

3 Roosevelt Warm Springs Rehabilitation, 6135 Roosevelt Highway, Warm Springs, GA 31830, USA

4 Grady Health System, Emory Department of Neurosurgery, Grady Memorial Hospital, 80 Jesse Hill Jr. Dr. SE, Atlanta, GA 30303, USA arthropathy (CSA)." [2, 3] CSA is characterized by progressive cartilaginous and bony destruction associated with loss of protective sensation to the spine.

In prior generations, CSA was primarily associated with tertiary syphilis, but in the modern era, the etiology of CSA is most often traumatic spinal cord injury (SCI) [2]. The location of CSA in SCI is typically below the level of injury, where there is loss of normal sensation. Although most commonly associated with traumatic SCI, CSA can be caused by any condition that impairs spinal sensory feedback, including hereditary insensitivity to pain, transverse myelitis, syringohydromyelia, diabetes, and others [3]. Persons with paraplegia with recurrent infections have been reported to be at increased risk for the development of CSA [4-6].

CSA typically involves destruction of anterior and posterior elements of the spine and is characterized by the "five Ds": debris, destruction, disorganization, dislocation (spondylolisthesis), and distention of tissues with mass formation [3]. Affected persons may remain asymptomatic or mildly symptomatic, even as striking bony destruction progresses in the spine. Symptoms reported are generally nonspecific and include changes in spasticity, pain, sitting 
balance, bladder function, and frequency of autonomic dysreflexia [7]. Physical exam findings may include spinal deformity (or gibbosity), audible crepitus, and changes in sensation or deep tendon reflexes. Spinal gibbosity, or abnormal swelling, is a consequence of the pathological process involving synovial effusion and hypertrophy, osteophytosis, and osteoclast hyperactivity [2].

A diagnosis of CSA is informed by radiographic findings, with identification of major disc and vertebral destruction, hypertrophic bone formation, and a soft tissue inflammatory "cocoon" surrounding the affected vertebral bodies [2, 8]. CT imaging reveals bony destruction and paravertebral bone formation, while MRI may better characterize fluid collections and soft tissue involvement surrounding the bony lesion. Biopsy is potentially required to rule out other causes of localized back pain and deformity, including infection and tumor $[7,8]$.

The following case report illustrates an unusual presentation of CSA, which has not previously been described in the literature.

\section{Case}

A 49-year-old male presented to the emergency room after an unwitnessed fall from 25 to 30 feet off a ladder while working as an electrician. He sustained a fracture/dislocation at T3-4 and T8-9, resulting in a T4 ASIA Impairment Scale (AIS) A.

Prior to his injury, past medical history was significant for hepatitis $\mathrm{C}$, alcohol abuse, methamphetamine drug abuse in remission, anxiety, and depression. He had no known allergies. He was functioning independently in all activities of daily living.

The patient underwent T2-T12 posterior spinal stabilization with an acute hospitalization course complicated by circulatory shock, acute respiratory failure, methicillinresistant Staphylococcus aureus pneumonia, a sacral pressure injury, and a complicated urinary tract infection. Following his injury, he was discharged to an acute rehabilitation hospital. He subsequently returned home, where he had assistance from his family members.

Seven months post-injury, he developed hardware failure and underwent extension of his hardware to L3 (Fig. 1). One year and 2 months after initial injury, he presented with an unusual swelling on his low back that had been increasing in size for about a month (Fig. 2). During this 1-month period, the patient had, additionally, noticed a decrease in his spasticity and concomitant new urinary incontinence between intermittent catheterizations, which he described as progressive "dribbling" of urine. Imaging revealed complete erosive destruction of the L3

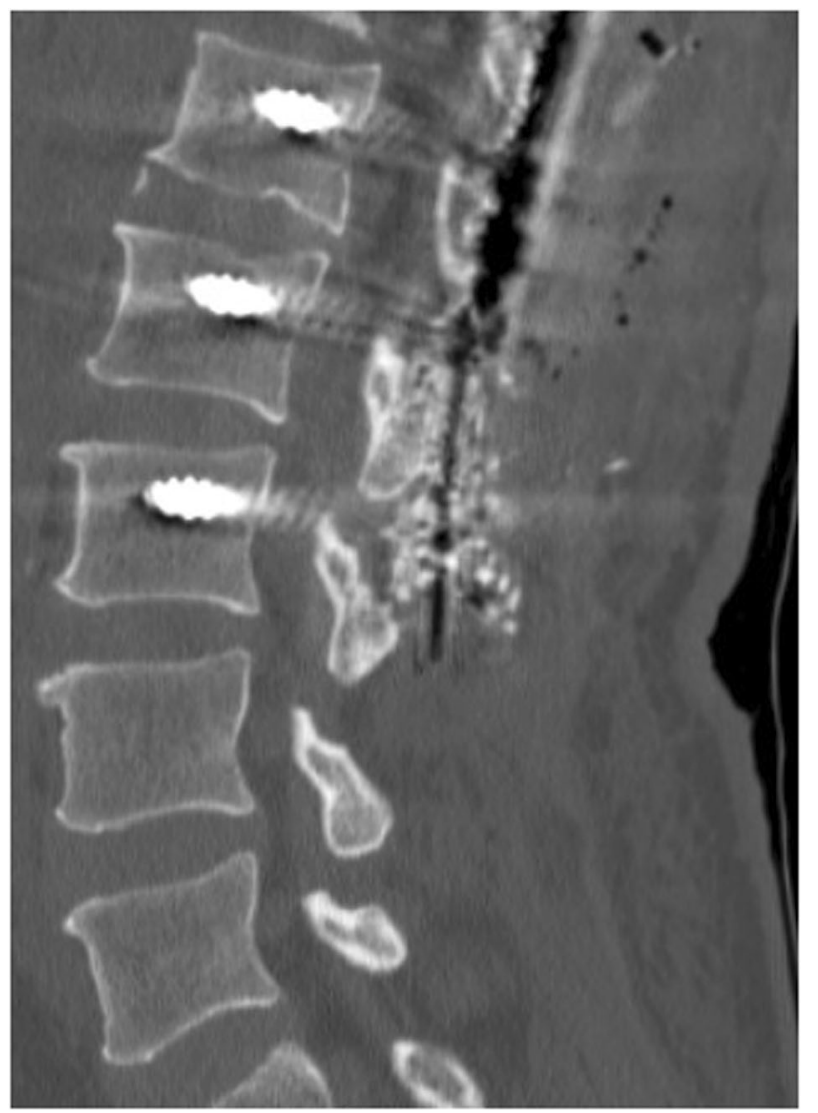

Fig. 1 Sagittal computed tomography (CT) scan of the lumbar spine without contrast following hardware extension demonstrating intact hardware and vertebral bodies

vertebral body, as well as partial destruction of L2 and L4 (Figs. 3-5). These imaging findings were found to be consistent with CSA.

Of note, the patient had been intentionally working on increasing forward flexion since his hardware extension. Shortly prior to his diagnosis of CSA, he had been happy to note a perceived increase in his spinal flexibility. Rather than represent a positive functional change, the observed increase in his lumbar flexibility was felt to be concerning for possible destruction of stabilizing elements of his lumbar spine, secondary to progressive CSA.

At the time of identification of the patient's CSA, he was being treated for a worsening stage IV pressure injury. The patient was treated by plastic surgery and physical medicine and rehabilitation providers for his pressure injury. When the patient's neurosurgeon was consulted regarding surgical management of CSA, the patient was deemed to be an inappropriate spine surgery candidate due to the risk of spinal hardware infection, given his active stage IV pressure injury. Surgical fixation of his spinal fracture was therefore deferred, pending sacral wound healing. 


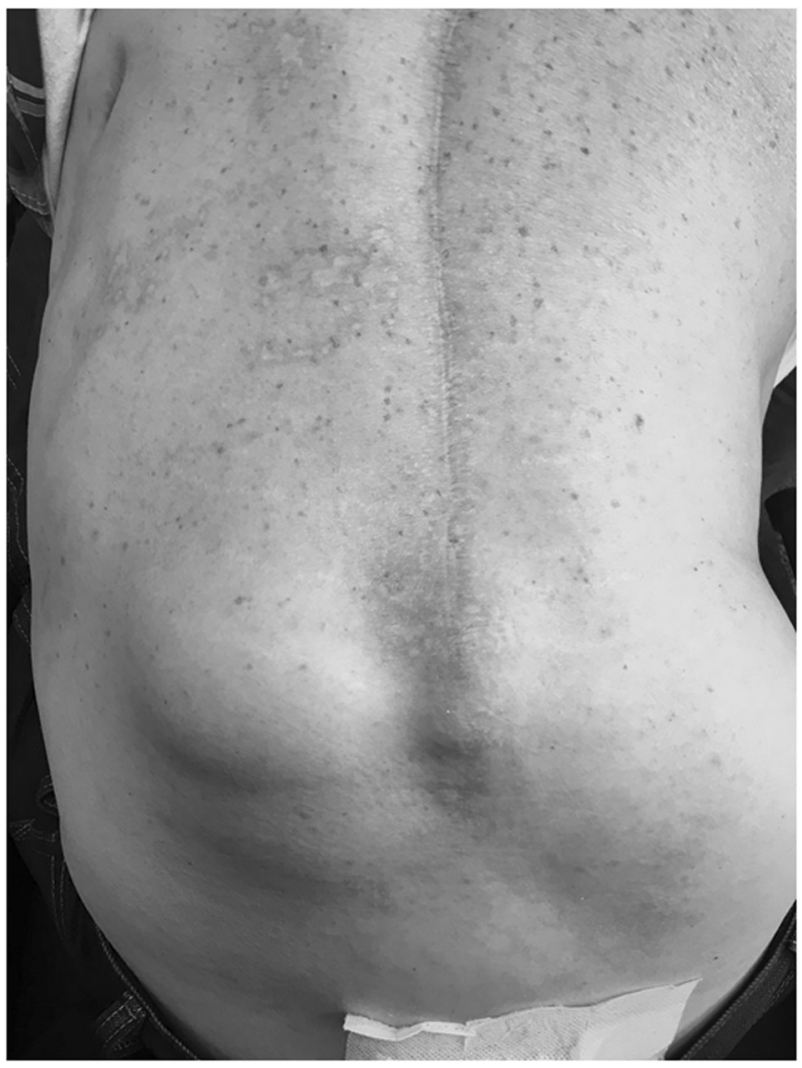

Fig. 2 Patient presented with complaint of a progressively enlarging swelling on his back over 1 month, consistent with spinal gibbosity

The patient was subsequently lost to follow-up and was not seen beyond the first year and a half after his injury by his physiatrists or neurosurgeons at the initial trauma hospital where he had previously received care.

Two years and 3 months after his initial injury, he was seen as an outpatient for wound care and plastic surgery evaluation. At that time, the patient's sacral wound had improved (but had not fully resolved) with conservative management. The patient was instructed to follow-up with his initial neurosurgeon for reconsideration of surgical management of his CSA. Following this plastic surgery visit, he was again lost to follow-up. The patient has not returned to his neurosurgeon or physiatrist for re-evaluation and there are no further medical records available to indicate his outcome.

\section{Discussion}

This case has unusual features that distinguish it from other published cases of CSA. One unusual feature is the very early presentation. In a comprehensive 2010 review by Barrey et al. of 109 patients with CSA, the mean time from onset of neurologic impairment to diagnosis of Charcot arthropathy was 17.3 years, plus or minus 10.8 years, with

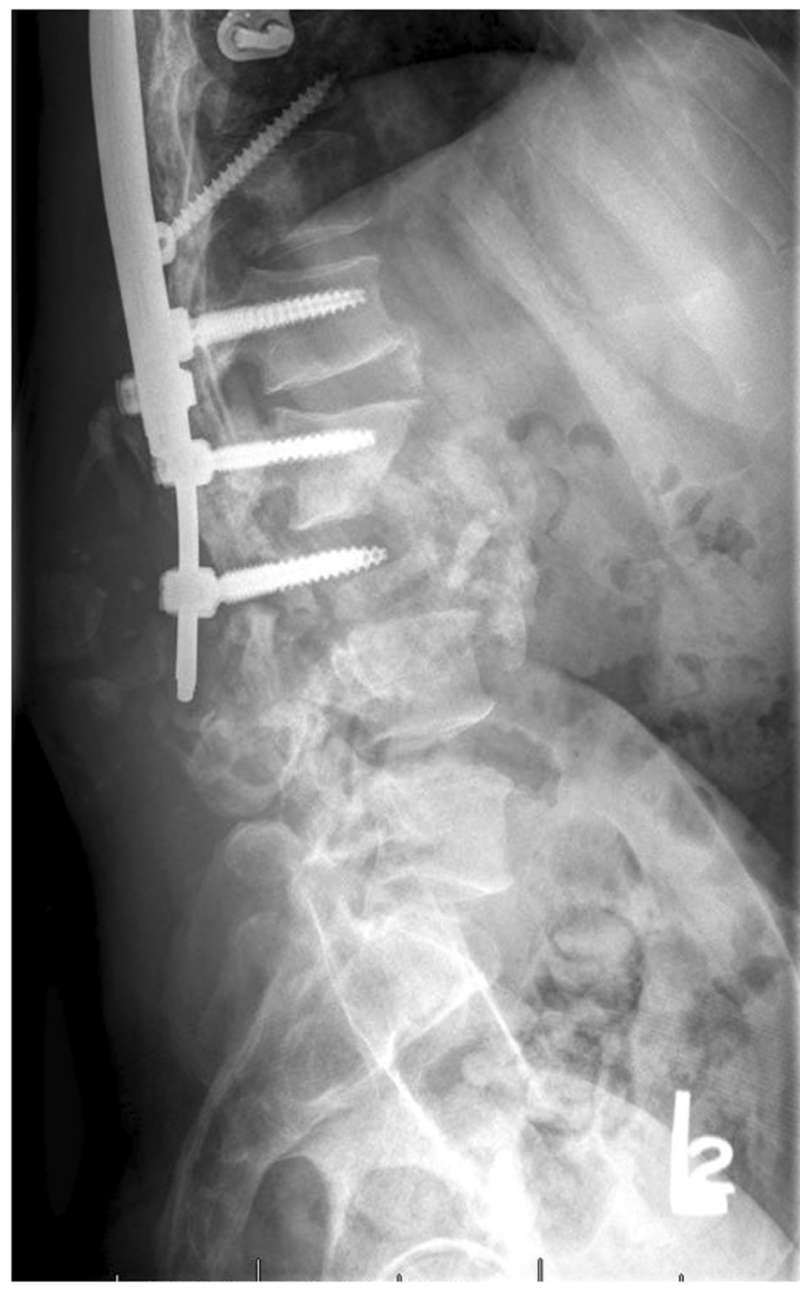

Fig. 3 Plain radiograph of the lumbar spine 1.2 years following injury. This image is notable for almost complete destruction of the L3 vertebral body and marked irregularity of the L 2 vertebral body. These changes were new compared with X-rays of lumbar spine 6 months prior. Differential included Charcot spinal arthropathy (CSA) vs. infectious osteomyelitis, with further imaging recommended by radiologist, specifically computed tomography (CT) or magnetic resonance imaging (MRI)

the shortest interval being 1.5 years [2]. Aebli described 28 patients who developed CSA a mean of 18.5 years after traumatic SCI (with a 95\% confidence interval (CI) of 12.5-20 years). The specific range from SCI was not specified [7]. Finally, Jacobs described 23 patients who were diagnosed with CSA after a mean of 19.6 years following SCI. This study revealed one case of CSA diagnosed sometime during the second year following traumatic SCI, although it is unclear at what point during that year the patient developed CSA [9]. No other cases were found in the literature revealing a shorter interval between SCI and CSA diagnosis. Thus, the case described here represents one of the earliest onsets of CSA found in the literature to date.

There were a number of clinical features concerning for the development of CSA in this case, despite the fact that 


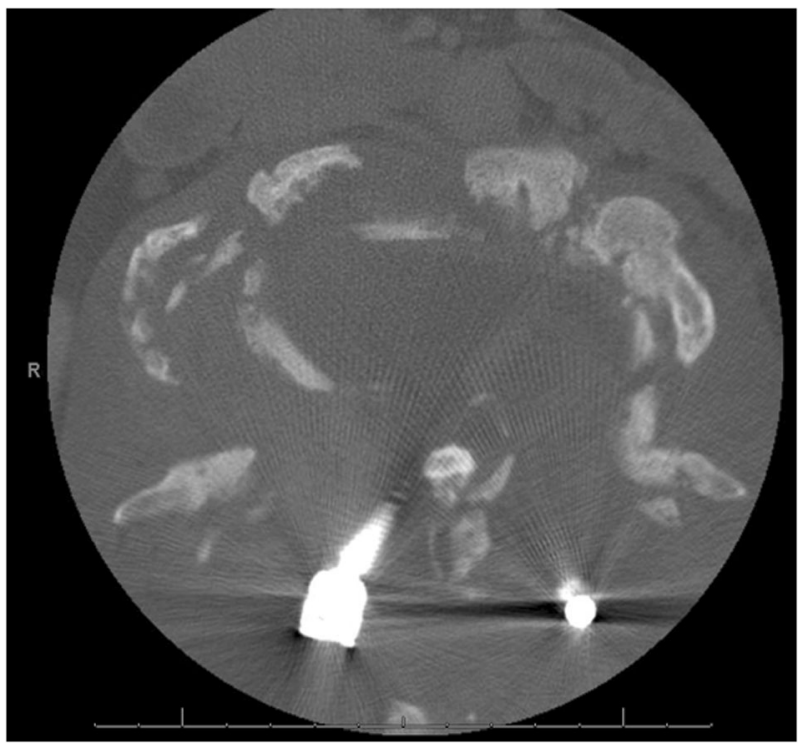

Fig. 4 Axial view computed tomography (CT) scan of the lumbar spine without contrast. Near complete destruction of L3 vertebral body is demonstrated, with residual bone fragments, extensive peripheral callus formation and dystrophic calcifications. Osseous destructive changes extend into the posterior elements and endplates of L2 and L4. There is almost complete obliteration of spinal cord at this level. Given preserved surrounding fat planes and lack of retroperitoneal lymphadenopathy, Charcot spinal arthropathy (CSA) was favored

this diagnosis would typically be suspected later in the clinical course following SCI than was seen here. Concerning features in this case pointing towards CSA included the increased spinal mobility, loss of spasticity, spinal swelling and deformity, and changes in bladder function. This case illustrates that when individuals present with these clinical features, CSA should be part of the differential regardless of the interval of time following their SCI. Although the patient was experiencing progressive neurologic changes, he did not follow-up with his neurosurgeon despite recommendations to do so, perhaps due to mistaken optimism regarding increased spinal mobility.

In appropriately selected patients with CSA, surgical fusion has the potential to reduce spinal deformity and stabilize the spine [9]. Alternatives to surgical stabilization are careful monitoring for continued neurologic decline and immobilization with a "body jacket" or orthotic [2]. There are individuals, however, for whom surgical intervention and other immobilizations may offer unacceptable risks, particularly for persons with complications of SCI, including severe sacral skin wounds.

The patient presented here had CSA with spinal changes identified at the L2-L4 levels. These levels are at the inferior pole of the surgical construct where he had previously undergone hardware extension. Reports from other authors regarding the development of CSA suggest that the development of CSA adjacent to prior instrumentation is

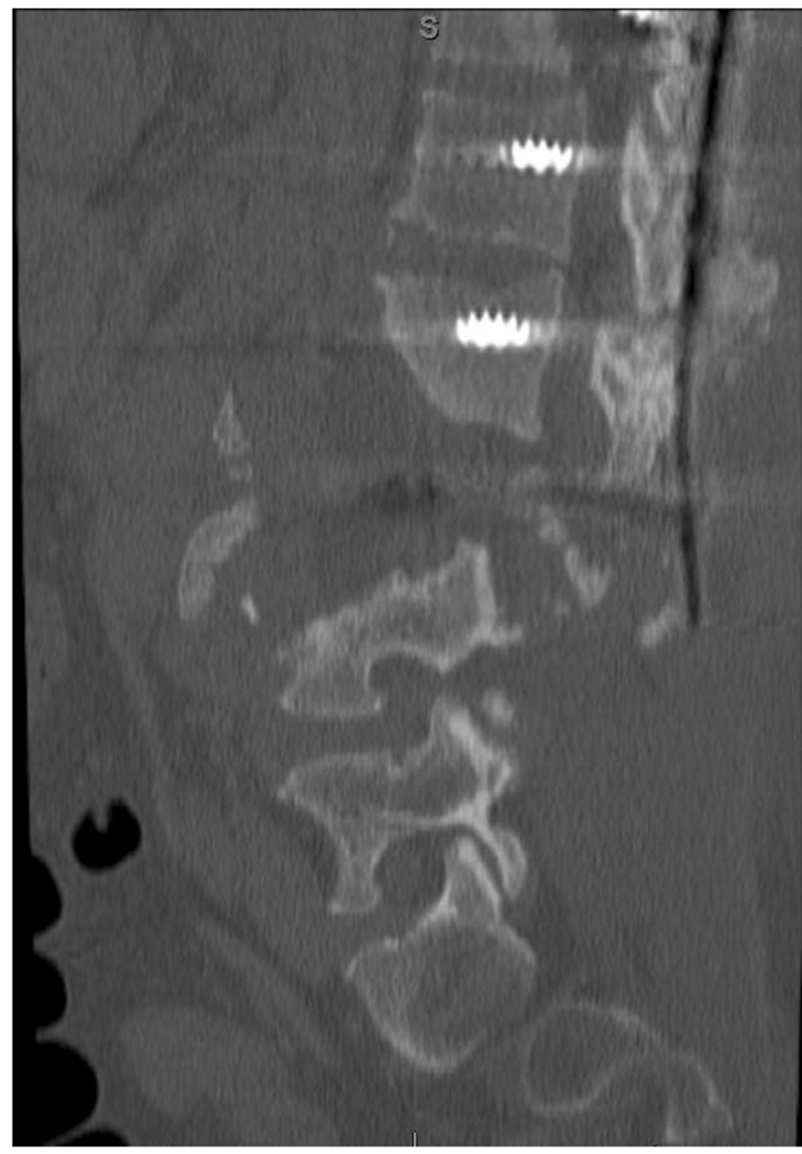

Fig. 5 Sagittal computed tomography (CT) scan of the lumbar spine without contrast. Again demonstrated is near complete destruction of L3 vertebral body with residual bone fragments and extensive peripheral callus formation and dystrophic calcifications. Osseous destructive changes extend into the posterior elements and endplates of L2 and L4. There is almost complete obliteration of the spinal cord at this level

typical [2]. When there is a history of prior spinal fixation or laminectomy, CSA typically develops at the location of the laminectomy or within two spinal segments of prior fusion $[3,10]$. Long fusions ending at the thoracolumbar junction appear to be at greater risk for complication by CSA [10]. Risk factors for CSA have been reported to include greater than five segment instrumentation, laminectomy, obesity, and excessive loading of the spine $[2,7,8]$.

An unusual feature of this case is that it was associated with intentional efforts to improve spinal range of motion through unsupervised forward flexion at home. The implication of the patient's attempts to improve forward flexion through an unsupervised exercise program is unclear. Some authors have suggested that the risk of early Charcot spine is greater in very active persons, although physical therapy has not been identified as a risk factor [2]. While upper trunk mobility, specifically lateral bending and rotation, has been reported to increase destructive forces on the lower spine and increase risk of development of CSA, to our knowledge 
there is no prior report of unsupervised intentional forward bending predisposing a patient to CSA [10]. Physical therapy is generally not contraindicated and has been suggested to be helpful for managing associated back pain [11]. Continual trauma from relatively minor injury, has, however, been identified as a contributing factor to CSA [1].

In evaluating this case, we recommend that exercise and therapy activities should be initiated in a supervised setting for patients with SCI. Any exercises that the patient is doing at home should be discussed at multiple points in time following the patient's injury.

\section{Conclusion}

CSA is an uncommon but important sequela of traumatic SCI and should not be missed given the potential risk of neurologic decline if untreated. This case highlights that individuals with relevant clinical features should be evaluated for CSA regardless of the time following their SCI. The implications of the patient's range of motion exercises are unclear. Future reports and studies on CSA should include evaluations of patients' exercise and therapy regimens.

\section{Compliance with ethical standards}

Conflict of interest The authors declare that they have no conflict of interest.

Publisher's note: Springer Nature remains neutral with regard to jurisdictional claims in published maps and institutional affiliations.

\section{References}

1. Gupta R. A short history of neuropathic arthropathy. Clin Orthop Relat Res. 1993;296:43-49.

2. Barrey C, Massourides H, Cotton F, Perrin G, Rode G. Charcot spine: two new case reports and a systematic review of 109 clinical cases from the literature. Ann Phys Rehabil Med. 2010;53:200-20.

3. Ledbetter L, Salzman K, Sanders RK, Shah L. Spinal neuroarthropathy: pathophysiology, clinical and imaging features, and differential diagnosis. Radiographics. 2016;36:783-99.

4. Goodwin RC, Ahmed AK, Abu-Bonsrah N, De la Garza-Ramos R, Petteys RJ, Sciubba DM. Charcot spinal arthropathy after spinal cord injury. Spine J. 2016;16:e545-e546.

5. Kalen V, Isono S, Cho C, Perkash I. Charcot arthopathy of the spine in long-standing paraplegia. Spine (Phila $\mathrm{Pa}$ 1976). 1987;12:42-47.

6. Grassner L, Geuther M, Mach O, Buhren V, Vastmans J, Maier D. Charcot spinal arthropathy: an increasing long-term sequel after spinal cord injury with no straightforward management. Spinal Cord Ser Cases. 2015;1:15022

7. Aebli N, Potzel T, Krebs J. Characteristics and surgical management of neuropathic (Charcot) spinal arthropathy after spinal cord injury. Spine J. 2014;14:884-91.

8. Viale R, Mary P, Tassin JL, Parker F, Guillaumat M. Charcot's disease of the spine: diagnosis and treatment. Spine (Phila PA 1976). 2005;30:e315-e322.

9. Jacobs WB, Bransford RJ, Bellabarba C, Chapman JR. Surgical management of Charcot spinal arthropathy: a single-center retrospective series highlighting the evolution of management. J Neurosurg Spine. 2012;17:422-31.

10. Standaert C, Cardenas D, Anderson P. Charcot spine as a late complication of traumatic spinal cord injury. Arch Phys Med Rehabil. 1997;78:221-5.

11. Moreu S, Lonjon G, Jameson R, Judet T, Garreau de Loubresse C. Do all Charcot spine require surgery? Orthop Traumatol Surg Res. 2014;11:779-84. 